\title{
HEART REMODELING, TREATMENT OF MYOCARDIAL INFARCTION WITH DIABETES MELLITUS 2ND TYPE AND HEART FAILURE
}

DOI: $10.36740 /$ WLek202006140

\author{
Maxim M. Potyazhenko ${ }^{1}$, Nadia 0. Lyulka ${ }^{1}$, Yulia A. Ostapchuk ${ }^{1,2}$ \\ 'UKRAINIAN MEDICAL STOMATOLOGICAL ACADEMY, POLTAVA, UKRAINE \\ 2POLTAVA REGIONAL CLINICAL MEDICAL CARDIOVASCULAR CENTER OF POLTAVA REGIONAL COUNCIL, POLTAVA, UKRAINE
}

\begin{abstract}
Scientific 42 articles were analyzed, which for analysis were taken from scientometric databases: Web of Science, Scopus, PubMed, Medline, on topics related to: heart remodeling, treatment of myocardial infarction with type 2 diabetes mellitus and heart failure. Analysis of current medical studies and the data obtained indicate both availability in the usage of new HF biological markers - suppressor of tumorigenesis 2 (ST2), and the efficiency in the use of antagonists of miniralocorticoid receptors and sodium-glucose linked transporter-2 inhibitors, which is complicated by HF and type 2 diabetes by slowing down the processes of LV myocardial remodeling, which promotes control of blood pressure and fluid volume and causes a decrease and gradual regression of $\mathrm{HF}$ regardless of its origin.
\end{abstract}

KEY WORDS: myocardial remodeling, acute myocardial infarction, heart failure, diabetes mellitus type 2

Wiad Lek. 2020;73(6):1284-1289

\section{INTRODUCTION}

Cardiovascular disease (CVD) is a leading cause of death worldwide $[1,2,3]$. According to the data of the World Health Organization, annually 18 million deaths are registered worldwide due to the pathology [2,3]. The countries of Europe and the USA show examples of successful struggle against this problem: within 20 years the mortality rate has decreased by more than $50 \%[1,3]$.

Diabetes mellitus (diabetes) is an important risk factor for acute myocardial infarction (MI). Although modern MI treatment has significantly favoured survival for both patients with and without diabetes. The presence of diabetes doubles twice as much the risk of mortality rate in the acute phase of MI and with long-term follow-up of patients. Higher risk of mortality among those with diabetes indicates a special need for better options in treating such patients and suggests that intensive medical treatment, long-term follow-up and strict control of other risk factors should be carefully performed and maintained $[4,5,6]$.

The risk of CVD (myocardial infarction, stroke, peripheral vascular disease) is twice as high in patients with type 2 diabetes compared with patients who do not have diabetes. Besides, CVD is a major cause of mortality among patients with type 2 diabetes [5].

\section{THE AIM}

The aim: review of current medical studies on diagnosing and treatment of complicated comorbid pathology: acute myocardial infarction (MI) complicated with heart failure
(HF) of type 2 diabetes patients, with structural and geometric changes in shape and function of the heart and the possibility of their pharmacological correction.

\section{MATERIALS AND METHODS}

42 scientific articles were analyzed, which for analysis were taken from scientometric databases: Web of Science, Scopus, PubMed, Medline, on topics related to: heart remodeling, treatment of myocardial infarction with type 2 diabetes mellitus and heart failure. Research methods: systematic approach, bibliosystematic analysis.

\section{REVIEW AND DISCUSSION}

The term "heart remodeling" was suggestesd by N.Sharp in the late 1970's to refer to heart structural and geometric changes after acute myocardial infarction (MI).

The main geometric types of left ventricular (LV) remodeling are related to the conditions under which they are developing. Pressure overload (aortic valve stenosis, hypertension) leads to increase in the number of sarcomeres and thickness of cardiomyocytes, wall thickness and formation of LV geometry concentric type. Volume overload (valve regurgitation) leads to the increase in the length of cardiomyocytes, decrease of wall thickness, increase in its volume and formation of LV geometry eccentric type. Myocardial infarction (MI) is a combination of pathogenetic mechanisms when stretching and enlarging the area of infarcted tissue leads to an increase in LV volume with overload and pressure of uninfected myocardial sections $[7,8]$. 
The myocyte is the main cardiac cell involved in the remodeling process. Other components involved in heart structural change are interstitium, fibroblasts, collagen, coronary vessels.

Pathogenetic postinfarction remodeling is a special type of left ventricular remodeling (LV) that develops in the result of increase in pre- and postload on the myocardium, increasing of the size of the left ventricle and hypertrophy of a healthy viable myocardium $[7,8]$. An increase in preload leads to a phenomenon of infarct expansion and increase in the volume of a healthy ventricular wall that is "resisted" by the infarcted ventricular wall [7]. The contraction of the heart muscle is not symmetrical because of the fact that necrotized segments lose their ability to contract [8]. As a result, the force generated by the unaffected myocardium during contraction is not counterbalanced by a similar retroactive force. As a consequence, the "infarcted" wall of the $\mathrm{LV}$ is stretched by the force of healthy walls contractions, which is not distributed evenly throughout the ventricle [7]. Thus, the situation is formed when the "infarcted" area must counteract a greater force of a healthy myocardium, which leads to asynchronous movement of the ventricular walls [7,9]. However, some affected segments may return to normal contractile capacity, though not immediately, but within months after the MI, it is the case of so-called "hibernated" myocardium (reperfusion injury reverse). However, under transmural myocardial lesions, some segments still remain dis-, hypo-, or akinetic, thereby causing permanent regional contractile dysfunction $[8,9]$. It has also been shown that in order to maintain a normal stroke volume with a reduced number of normally functioning segments, a healthy myocardium is forced to produce more pressure required to increase the postload on a healthy myocardium [7].

Myocardial remodeling is commonly developping in patients with ST segment elevation myocardial infarction (STEMI), where the scar is transmural. There are many predictors of this phenomenon and they can be evaluated by a variety of visualizing (ultrasound, CT, MRI) and laboratory techniques. The best predictor of remodeling is undoubtedly the size of infarction, which can be measured by the percentage ration to the mass of myocardial mass [10]. During acute MI, serum troponin I and MB fraction of creatine phosphokinase correlate positively with MI size; their very high levels may indicate an increase in the volume of cameras, as well as a decrease in global and segmental contractility $[9,10]$.

The development of postinfarction LV remodeling puts the patient at risk of sudden cardiac death (for instance, due to the development of fatal arrhythmias) [11]. Qualitative disorders of LV geometry and myocardial structure, together with the increased process of fibrosing of the heart walls, may lead to possible extraconductive transmission, which can contribute to the development of arrhythmia by the mechanism of micro- and macro-re-entry $[7,11]$. It is also proved that structural changes can increase the electric automatism of cardiomyocytes [11]. There is sufficient evidence that eccentric LV hypertrophy is associated with a threefold increase in the risk of major cardiovascular events, including sudden death, recurrent heart attack, heart failure, stroke $[7,8,11]$. Scar expansion often results in pronounced dilatation of the ventricle and aneurysm, the wall of which consists of myocardial segments, which are usually akinetic [10].Slow blood flow in the aneurysm can lead to thrombus formation. LV thrombosis is usually asymptomatic but is often associated with a high risk of systemic thromboembolism, including transient ischemic attacks and strokes $[8,10]$.

Thus, taking into account things mentioned above, it should be emphasized that myocardial remodeling occurs by restructuring of components - cardiomyocytes, their vessels and the intracellular matrix $[7,10]$. Normally, the wall of the ventricle consists of three layers of fibres: longitudinal, circular, and helical, which rotate from subepicardial to subendocardial layer ranging almost to 180 degrees. In a healthy myocardium, the thickness of all three layers is identical. $[7,8]$. All the fibers consist of tightly grouped cardiomyocytes. The layers of the fibers are separated by furrows. The laminar architecture of the myocardium is due to a complex system of extracellular matrix proteins and fibrillar collagen. Approximately $85 \%$ of all cardiac collagen is type I collagen, which has contractile force [10]. Type III collagen occupies $11 \%$; it is formed as thin filaments and provides elasticity of the walls. Based on the morphological characteristics, the entire cardiac matrix can be divided into three components: epic, peri, and endomyzes [12] Epimysium is located on the endocardial and epicardial surfaces and supports meso- and endothelial cells. The perimysium surrounds the muscle fibers and therefore connects the muscle fibers together. The endomysium diverge from the perimysium and surrounds separate muscle fibers. Endomysium binds muscle fibers and supply nourishment $[7,12]$. Postinfarction magnetic resonance mapping shows the disappearance of subendocardial fibers and hypertrophy of the subepicardial layer in the affected segments. Zones without muscle fibers refer to a postinfarction scar. Cardiomyocytes at those places are replaced by collagen $[10,12]$. Hypertrophied cardiomyocytes are longer than other normal heart cells and surround the scar area, although they may also be present in remote areas of the myocardium. This type of hypertrophy is called eccentric. In this case, the deterioration of the ventricle due to volume overload is observed $[9,10]$. Due to the extremely limited regenerative supply, hypertrophy that occurs in the postinfarction period is accompanied by an increase in the extracellular matrix (ECM), which is mostly composed of collagen fibers $[7,10]$. It is due to the increased activity of cardiac fibroblasts, which are activated by various fibrogenic mediators $[8,9]$. Increase of the load on the walls in infarcted myocardium leads to thinning of the affected wall by Laplace law and activates the synthesis of collagen by fibroblasts. Extension of the ECM reduces the load on cardiomyocytes but impairs their contractile function [13]. As a result, there appeares negative correlation between ECM volume and left ventricular (LV) ejection fraction (EF) $[12,13]$. There is also evidence that ECM volume correlates positively with mortality $[10,13]$. 
The term "myocardial remodeling" was suggested to characterize the response of the myocardium to the development and progressing of acute myocardial infarction to chronic heart failure (HF) [14]. Regardless the fact that remodeling is most commonly associated with cardiovascular events, its effects are directly dependent on the size of the necrosis $[8,15]$. Postinfarction remodeling develops in $30 \%$ of patients with MI, and as it is a predictor of $\mathrm{HF}$, this phenomenon is considered prognostically unfavorable $[8,12,14]$. Low-EF HF affects more than 20 million people worldwide [15]. Left ventricular HF within the patients older than 65 is the leading cause of hospitalization. Recently, It was noticed recently, that there exists a different category of HF - HF with functioning EF. $[10,12]$. Such kind of patients is represented by a completely different phenotype with biochemical features different from traditional ones. Therefore, it became necessary to identify the characteristics and predictors of both negative myocardial remodeling and recovering myocardial function of patients with HF after AMI. [12,13]. Clinical symptoms of HF are also the consequence of cardiac remodeling, which includes damage of cardiac cells and extracellular matrix [7] followed with cell hypertrophy, apoptosis and necrosis of myocytes, activation and proliferation of fibroblasts, which ultimately leads to fibrosis and myocardial dysfunction.

Among patients with HF, the evolution of the disease and response to therapy differ as well as the consequences that depend on the cause of the HF, the pathophysiological characteristics (systolic or diastolic dysfunction), the severity of HF, the speed of its progression. [16,17]. In diabetes, postinfarction impairment of heart pumping function is usually associated with a subclinical disturbance of LV function that occurred before MI $[8,17]$. In the stage of acute ischemia, patients have "compensatory" hyperkinesia of the myocardium unaffected areas; this hyperkinesia can normalize the overall ejection fraction and correlate with hemodynamic status and survival of patients [10]. A number of studies show that after MI LVEF [7] and regional EF of intact myocardium [17,18] are lower in diabetes than without it. The results of early angiography in TAMI (Thrombolysis and Angioplasty in Myocardial Infarction) studies show that the function of the ventricular myocardium intact sections in diabetes is worse than when it is absent $[18,19,20]$.

Speaking about pathogenic processes of myocardial remodeling, a special group of patients, namely, the individuals with high and very high risk of fatal cardiovascular events, individuals with MI and concomitant type 2 diabetes $[4,18,20]$ should be of special interest. Chronic hyperglycemia promotes morphofunctional myocardial remodeling, which leads to diabetic cardiomyopathy (DCM). DCM is described as HF with functioning LVEF, the first distinguishing feature being diastolic dysfunction (DD) in combination with concentric cardiac hypertrophy. [4,20,21]. DCM initially reveals as a decrease in the elongation of the left ventricle on the background of normal systolic function $[21,22]$. Asymptomatic disorders of diastolic function develop in $27-69 \%$ of patients with diabetes mellitus (microcirculatory disorders in this case are either absent or poorly expressed) [22,23]. Concomitant arterial hypertension develops in diabetic patients about twice as often as in general population [24], and more grave cardiomyopathy were noticed within them [22]. Hypertension leads to LV hypertrophy and helps to reduce its elongation $[22,24]$. However, dysfunction of diastolic relaxation in diabetes patients was also registed in the absence of concomitant hypertension [23]. Evident signs of impaired systolic function usually occur with prolonged diabetes, severe microangiopathy or concomitant arterial hypertension [24]. Thus, DCM, occurring subclinically without atherosclerotic lesions of large vessels, reduces the compensatory capacity of the myocardium, which is not damaged by heart attack. When the degree of myocardial necrosis is the same, the clinical picture depends on the severity of diabetic cardiomyopathy $[22,24]$.

Because of the peculiarities of the pathophysiological processes that lead to the development of HF as a result of myocardial remodeling after MI, there occurred the need to stratify the risk of prognosticaliy unfavorable myocardial changes based on certain parameters. Besides clinical signs which refer to existing assessment prognostic scales, recently biomarkers are also used [24,25].

Biomarkers can be useful for diagnosing proceses, choice of the way of treatment, in monitoring treatment efficacy and predicting outcomes of the disease due to their high sensitivity and specificity in case of thorough laboratory controle. Within the patients with HF, the evolution of the disease and the response to therapy differ as well as the consequences that depends on the cause of the HF, pathophysiological characteristics (systolic or diastolic dysfunction), the severity of the HF, the rate of its progression $[25,26,27]$.

During two last decades, the prognosis and clinical approaches to the treatment of patients with heart failure have been based on the study of biomarkers such as B-type brain natriuretic peptide (BNP) and N-terminal brain natriuretic peptide fragment (NT-proBNP). NT-proBNP is a highly sensitive to ischemia biological marker that is secreted in the myocardial lesion and reflects profile of myocardial structure and function disorders [25,27]. In addition to mechanical stretching of the ventricles, there are probably other mechanisms that stimulate the formation of NT-proBNP, namely: ischemia of different localization, rhythm disturbance, myocardial hypertrophy, endothelial dysfunction $[27,28]$. However, as an indicator of adverse course of myocardial infarction, NT-proBNP has low diagnostic specificity and sensitivity. Besides, in some cases, especially in the presence of concomitant pathology, the data of the biomarker was not always reliable because such indicators as age, sex, renal function, body mass index, thyroid function and anemia can effect it. [27,29]. The amount of NT-proBNP in the blood may also change in case of a infectious diseases. Therefore, there is now a search for new biomarkers capable of detecting HF at an earlier stage, assessing the prognosis for the patient, determining the severity of the disease, possible consequences 
and response to the treatment. One of the most promising among the new biomarkers is the suppressor of tumorigenesis 2 (ST2) [26,30,31]. It was discovered in 1989, but only in 2002 Weinberg E.O. et al. reported that it can be secreted by cardiomyocytes in response to biomechanical stress of the myocardium, that attracts attention of researchers to the role of this marker in the cardiovascular system [29,32].

ST2 is a member of the interleukin-1 receptor family and exists in two different forms: transmembrane isoform (ST2L) and soluble isoform (ST2). ST2 is an interleukin-33 (IL-33) receptor, which is an IL-1-like cytokine secreting with living cells in response to their damage. The main function of ST2 is the potentiation of interleukin 33 (IL-33) effects, which exerts its effect by binding to the isoforms of the transmembrane receptor ST2L.It was proved experimentally that interaction of IL-33 and ST2L is cardioprotective and reduce myocardial fibrosis, cardiomyocyte hypertrophy, apoptosis, and improve cardiac muscle function. [33]. This cardioprotective effect is performed solely through the ST2L receptor and not through the soluble receptor. The IL-33 / ST2 system is activated in cardiomyocytes and fibroblasts in response to myocardial damage, sST2 binds to IL-33 that competes with ST2L. The interaction of this soluble receptor with IL-33 blocks the IL-33 / ST2L system and, as a result, eliminates the cardioprotective effects described above, and sST2 is considered to be a bait receptor [34]. Thus, the ST2 system acts not only as a mediator of IL-33 function in its transmembrane isoform ST2L, but also as an inhibitor of IL-33 due to its soluble sST2 isoform [30,32]. However, a sharp increase in ST2 levels in the damage is accompanied by suppression of the favorable antihypertrophic effects of IL-33 [33]. Therefore, the reasout of ST2 in MI may have prognostic value for patients, as it will allow to evaluate the course of the acute period and the possibility of complications.

The major sources of sST2 are cardiac fibroblasts and cardiomyocytes, which release it in response to biomechanical stress or damage, but there are also non-myocardial sources of soluble ST2. Such sources of sST2 are endothelial cells from both macrovascular (aortic and coronary) and cardiac microvascular systems $[31,32]$.

There is no currently a convincing evidence for the treatment of HF with functioning LV EF, thus, interest to detecting biomarkers that reflect multiple mechanisms associated with the pathogenesis of syndrome of this $\mathrm{HF}$ variant is growing [25]. Bhardwaj A. and Januzzi J.'s review of the role of ST2 as a new biomarker of HF compares the prognostic value of NT-proBNP and ST2 [26,27,32].

Considerable attention is paid to comorbid conditions, especially with coronary heart disease in combination with diabetes. The results of epidemiological studies indicate that the risk of coronary complications in patients with diabetes without coronary heart disease can be compared with the risk in patients who already have clinical manifestations of coronary heart disease $[18,29]$. Such patients may have no clinical symptoms or signs of moderate diastolic dysfunction at an early stage, and shortness of breath, fatigue, weakness and swelling of shin may develop in the course of progressing pathology [21,22]. Interstitial and perivascular fibrosis is a histological indicator of diabetic cardiomyopathy, and the degree of fibrosis correlates with myocardial hypertrophy $[21,22]$. Taking into account the pathophysiological indicators, echocardiographic changes, and serological biomarkers, one can assume that the initial stage of DCM is characterized with mixed hypertrophic and restrictive phenotype. At this stage, microangiopathy and hypertension promote the development of DD, with the progression of which the systolic function is impairing in the result of dilatation, fibrosis, micro- and macroangiopathy $[21,22,24]$.

Special researches, when analyzed, vividly prove that applying the principles of pharmacological impact on myocardial remodeling is appropriate and may be a useful strategy for the treatment of patients with STEMI / NSTEMI. Usage of pharmacological devices at an early stage to effects myocardial remodeling is necessary to prevent possible new cardiovascular events, accelerate the processes of restoring normal functioning of the cardiovascular system, improve rehabilitation measures and reduce the probability of death.

It is clear that the main way to reduce the fatal and non-fatal consequences of HF is primary prevention $[20,35,36$,$] .$

In the pathogenetic mechanisms of postinfarction LV myocardial remodeling a significant role belongs to the fact how active the angiotensin-aldosterone system is. Numerous studies indicate that aldosterone impairs the contractility and metabolic functions of ischemic myocardium, increases systemic vascular resistance, and enhances the vasoconstrictor effect of angiotensin II in the coronary arteries [37]. Activation of mineralocorticoid receptors leads to myocardial fibrosis, inflammation, ruining of cardiomyocyte, and LV hypertrophy [38]. It was reaffirmed that mineralocorticoid receptor antagonists (AMCR), which are represented by the nonselective agent spironolactone [44] and selective eplerenone [39], favour the survival of patients with HF and MI with LV systolic dysfunction. This clinical bonus are associated with improved LV remodeling and reduced myocardial fibrosis [38]. Spironolactone and eplerenone differ in their molecular structure, pharmacodynamics and pleiotropic effects $[37,38,39,40]$, however, the significant clinical differences between the two agents are not clearly defined and their usage in case of preserved LV EF that functios, requires further studies.

According to multicenter studies, AMCRs affect the level of fibrosis markers. It is important to note that the development of myocardial fibrosis is not only a factor contributing to the progression of HF, but also causes electrical instability of the myocardium, conductivity disorders, which in its turn leads to an increased risk of ventricular arrhythmias and SCD [11].

DM is one of the predictors of HF [24]. This is especially true for HF with normally functioning LV EF [21]. Intracellular metabolic disorders and increased oxidative stress due to hyperglycemia, insulin resistance and chronic inflammation are pathogenic mechanisms that affect LV diastolic dysfunction against the backgroung of type 2 
diabetes. These mechanisms lead to structural changes in the heart, such as LV hypertrophy and interstitial fibrosis, which lead to HF $[8,20,21]$.

The risk of death in combination of MI with diabetes is much higher than in the acute period of MI, or when lasting for several years $[5,18]$. HF of such comorbid patients is more pronounced and there exists a higher probability of recurrent MI [5,21].

In current clinical practice, type 2 diabetes therapy should aim not only at achieving glycemic control but also at influencing other modified CVD risk factors, including hypertension, overweight, frequent hypoglycemia, etc. [41]. Many antipyretics have a high potential for reducing glycated hemoglobin (HbA1c) level. Taking into account complex pathophysiological bindings between diabetes mellitus, obesity, arterial hypertension, and atherosclerosis it is very important that all glycemia controlling camicals, available and those new ones that just appear should be able not only control glycemial, but also effect the factors of $\mathrm{CD}$ risk in patients with type 2 diabetes $[4,18]$. Increased reabsorption of glucose in the kidneys is an important pathogenetic mechanism that supports chronic hyperglycemia in diabetes mellitus. About 90\% of glucose is reabsorbed in the proximal renal tubule by sodium-glucose transporters of the second type (SGLT-2), and the remaining $10 \%$ by means of glucose transporters of the first type (SGLT-1) located distally. In type 2 diabetes, the activity of SGLT-2 increases, the capacity of the renal glucose transport increases, and, as a consequence, the renal glucose threshold increases $[18,42]$. Glyphlozins inhibit SGLT-2, which causes a decrease in sodium and glucose reabsorption from the lumen of the proximal renal tubule and leads to glucosuria. In the course of retrospective analysis of the EMPA-REG OUTCOME, which covered adult patients with type 2 diabetes and confirmed CVD, the beneficial effect of SGLT-2 on the cardiovascular system was evaluated. The analysis performed showed that SGLT-2 determined cardiovascular benefits, namely the reduction of cardiovascular mortality and hospitalization for HF in patients with diabetes mellitus and CVD based on an assessment of cardiovascular risk factors.

\section{CONCLUSIONS}

According to the analysis of scientific literature, these data indicate the prospect of using new biological markers - ST2, for the early diagnosis of heart failure (HF). However, the use of mineralocorticoid receptor antagonists and inhibitors of type 2 sodium glucose cotransporter in the treatment of patients with acute myocardial infarction complicated by HF and concomitant type 2 diabetes slows down gradual regression of manifestations of $\mathrm{HF}$, regardless of its origin.

\section{REFERENCES}

1. Berger J.S., Jordan C.0., Lloyd-Jones D., Blumental R. Screening for cardiovascular risk in asymptomatic patients. J.Am.Coll.Card.; 2010; 55: 1169-1177.
2. Go, A. S. Heart disease and stroke statistics-2013 update: a report from the American Heart Association. Circulation ; 2013; 127: 6-245.

3. Yancy C.W., Jessup M., Bozkurt B. et al. American College of Cardiology Foundation; American Heart Association Task Force on Practice Guidelines. 2013 ACCF/AHA Guideline for the management of heart failure: a report of the American College of Cardiology Foundation/ American Heart Association Task Force on Practice Guidelines. J Am Coll Cardiol. 2013;62(16):e147-239. doi:10.1016/j.jacc.2013.05.019/.

4. Dauris M., Targher G., Laroche C. et al. For the ESC-HFA Heart Failure Long Term Registry. Association detween diabetes and 1-year adverse clinical outcomes in multinational cohort of patients with chronic heartb failure: results from the ESC-HFA Heart Failure Long Term Registry. Diabetes Care; 2017; 40: 671-678.

5. Lehrke M., Marx N. Diabetes Mellitus and Heart Failure. Am J. Med. 2017;130(6S):S40-S50. doi: 10.1016/j.amjmed.2017.04.010.

6. Shah A.D., Langenberg C., Rapsomaniki E. et al. Type 2 diabetes and incidence of cardiovascular diseases: a cohort study in 1.9 million people. Lancet Diabetes Endocrinol. 2015;3:105-13.

7. Galli A., Lombardi F. Postinfarct left ventricular remodeling: a prevailing cause of heart failure. Cardiology research and practice, vol.2016, 12p.

8. Heusch G., Libby P., Gersh B., et al. Cardiovascular remodeling in coronary artery disease and heart failure. Lancet; 2014; 383:1933-43

9. Chan W., Duffy S., White D., et al., Acute left ventricular remodeling following myocardial infarction: coupling of regional healing with remote extracellular matrix expansion, JACC: Cardiovascular Imaging, 2012;5(9): 884-893

10. Downey J.M., Cohen M.V. Reducing infarct size in the setting of acute myocardial infarction. Progress in Cardiovascular Diseases. 2006;48:363-376

11. Bauer A. Improved Stratification of Autonomic Regulation for risk prediction in post-infarction patients with preserved left ventricular function (ISAR- RISK) Eur. Heart. J.; 2009;30:576-583.

12. Borlaug B.A. The pathophysiology of heart failure with preserved ejection frection. Nat. Rev. Cardiol.2014; 11: 507-515.

13. Seropian I.M., Toldo S., Van Tassell B.W., Abbate A. Anti-inflammatory strategies for ventricular remodeling following ST-segment elevation acute myocardial infarction. J Am Coll Cardiol. 2014;63(16):1593-603. doi: 10.1016/j.jacc.2014.01.014.

14. Perk J., Bucker G., Gohlke H. European Guidelines on cardiovascular disease prevention in clinical practice (version 2012). Eur Heart J.; 2012; Vol. 33 (13): 1635-1701.

15. Townsend N., Wilson L., Bhatnagar P. et al. Cardiovascular disease in Europe: epidemiological update 2016. Eur Heart J 2016;37(42):32323245.

16. Braunwald E., Libby P., Bonow R. Heart diseases. A textbook of cardiovascular medicine. NY: Saunders.; 2009; 2300.

17. De Boer R.A., Yu L., Van Veldhuisen D.J. et al (2010) Galectin-3 in cardiac remodeling and heart failure. Curr Heart Fail Rep 7(1):1-8

18. Type 2 Diabetes Mellitus and Heart Failure. A Scientific Statement From American Heart Association and Heart Failure Socieety of America; 2019; 139:00-00. DOI:10.1161/CIR.0000000000000691.

19. Frassdorf J., De Hert S., Schlack W. Anaesthesia and myocardial ischaemia/reperfusion injury. Br J Anaesth.; 2009; 103(1):89-98.

20. Johanson I., Dahlstrom U., Edner M. Prognosis implication of type 2 diabetes mellitus in ischemic an non ischemic heart failure. J Am Coll. 2016; 68: 1404-1416.

21. Lam C.S. Diabetic cardiomyopathy: An expression of stage B heart failure with preserved ejection fraction. Diabetes and Vascular Disease Research. 2015;12(4):234-8 
22. Chong C-R., Clarke K., Levelt E. Metabolic remodelling in diabetic cardiomyopathy. Cardiovasc. Res. 2017;113(4):422-430. doi:10.1093/ $\mathrm{cvr} / \mathrm{cvx} 018$.

23. Miki T., Yuda S., Kouzu H., Miura T. Diabetic cardiomyopathy: pathophysiology and clinical features. Heart Fail Rev. 2013;18(2):14966. doi: 10.1007/s10741-012-9313-3.

24. Nichols M., Townsend N., Scarboroug P. Cardiovascular disease in Europe 2014: epidemiological update. Eur. Heart J.; 2014; 35 (42): 2929.

25. Biomarkers Definitions Working Group. Biomarkers and surrogate end-points: preferred definitions and conceptual framework, Clin. Pharmacol. Ther. 2001;69: 89-95.

26. Lin Y-H., Zhang R-C., Hou L-B. et al. Distribution and clinical association of plasma soluble ST2 during the development of type 2 diabetes. Diabetes Research and Clinical Practice. 2016;118:140-5.

27. Fu S., Ping P., Zhu Q. et al. Brain Natriuretic Peptide and Its Biochemical, Analytical, and Clinical Issues in Heart Failure: A Narrative Review. Front Physiol. 2018; 9: 692.doi: 10.3389/fphys.2018.00692.

28. Kalsmith B.M. Role of the Brain Natriuretic Peptide in Heart Failure Management. Circulation: Heart Failure. 2009; 2:379. doi: 10.1161 / CIRCHEARTFAILURE.108.816264.

29. Lichtenauer M., Jirak P., Wernly B. et al. A comparative analysis of novel cardiovascular biomarkers in patients with chronic heart failure. Eur J Intern Med. 2017;44:31-38. doi: 10.1016/j.ejim.2017.05.027.

30. Ky B., French B., McCloskey K. et al. Highsensitivity ST2 for prediction of adverse outcomes in chronic heart failure. Circ Heart Fail 2011;4:180-7.

31. Weir R.A., Miller A.M., Murphy G.E. et al. Serum soluble ST2: a potential novel mediator in left ventricular and infarct remodeling after acute myocardial infarction. J Am Coll Cardiol. 2010;55(3):243-50. doi: 10.1016/j.jacc.2009.08.047.

32. Bhardwaj A., Januzzi J.L. ST2: a novel biomarker for heart failure. Expert Rev. Mol. Diagn. 2010;10(4):459-64.

33. Sanada S., Hakuno D., Higgins L.J. et al. IL-33 and ST2 comprise a critical biochemically induced and cardioprotective signaling system. J. Clin. Invest. 2007;117:1538-49.

34. Seki K., Sanada S., Kudinova A.Y. et al. Interleukin-33 prevents apoptosis and improves survival after experimental myocardial infarction through ST2 signaling. Circ. Heart Fail. 2009;2 684-91.

35. Bulluck H. et al. Reducing myocardial infarct size: challenges and future opportunities. Heart.2016;102:341-348

36. Marwick T., Ritchie R., Shaw J., Kaye D. Implication of underlying mechanisms for recognition and management of diabetic cardiomyopathy. J Am Cardiol. 2018;71:339-351.

37. Talman V., Ruskoaho H. Cardiac fibrosis in myocardial infarction-from repair and remodeling to regeneration. Cell Tissue Res. 2016;365(3):56381. doi: 10.1007/s00441-016-2431-9.
38. Vizzardi E., Regazzoni V., Caretta G. et al. Mineralocorticoid receptor antagonist in heart failure: Past, present and future perspectives. Int J Cardiol Heart Vessel. 2014;3: 6-14. doi: 10.1016/j.jijchv.2014.03.005

39. Wu C.T., Wang Z.H., Li Z.Q., Wang LF. Effect of spironolactone on cardiac remodeling after acute myocardial infarction. World J Emerg Med. 2013;4(1):48-53. doi: 10.5847/wjem.j.issn.1920-8642.2013.01.009.

40. Potyazhenko M.M., Skripnik I.M., Vakulenko K. Et al. Efektyvnist' antahonistiv al'dosteronu u khvorykh na infarkt miokarda, uskladnenyy hostroyu livoshlunochkovoyu nedostatnistyu z zberezhenoyu systolichnoyu funktsiyeyu livoho shlunochka. [Efficiency of aldosteron antagonists for patients with myocardial infarction, complicated with acute leftventricle insufficiency] Артеріальна гіпертензія. 2018; 2(58):52-56. (in Ukrainian).

41. Gielen S., Landmesser U. The year in cardiology 2013: cardiovascular diseases prevention. Eur. Heart J.; 2014; 35(5): 307-312.

42. Gilca G-E., Stefanescu G., Badulescu 0. et al. Diabetic Cardiomyopathy: Current Approach and Potential Diagnostic and Therapeutic Targets. J Diabetes Res. 2017;2017:1310265. doi: 10.1155/2017/1310265.

Research topic: "Features of the course of cardiovascular pathology in patients of different age categories, depending on the presence of components of metabolic syndrome and comorbid conditions, ways of correction of revealed disorders and prevention". State registration number: 0119U102864.

\section{ORCID and contributorship:}

Maxim M. Potyazhenko - 0000-0001-9398-1378 ${ }^{E, F}$

Nadia O. Lyulka - 0000-0002-2301-5374 A,F

Yulia A. Ostapchuk - 0000-0002-4833-6510 B,D

\section{Conflict of interest:}

The Authors declare no conflict of interest.

\section{CORRESPONDING AUTHOR Yulia A. Ostapchuk}

Department of Internal Diseases and Emergency Medicine Ukrainian Medical Dental Academy

St. Shevchenko, 23, 36024 Poltava, Ukraine

tel: +380663338059

e-mail:yulia_0stapchuk.91@ukr.net

Received: 22.02 .2020

Accepted: 30.04 .2020 